\title{
Contravariant Boussinesq equations for the simulation of wave transformation, breaking and run-up
}

\author{
F. Gallerano, G. Cannata, O. De Gaudenzi \& S. Scarpone \\ Department of Civil, Constructional and Environmental Engineering, \\ University of Rome 'La Sapienza', Italy
}

\begin{abstract}
We propose an integral form of the fully non-linear Boussinesq equations in contravariant formulation, in which Christoffel symbols are avoided, in order to simulate wave transformation phenomena, wave breaking and near shore currents in computational domains representing the complex morphology of real coastal regions. The motion equations retain the term related to the approximation to the second order of the vertical vorticity. A new Upwind Weighted Essentially Non-Oscillatory scheme for the solution of the fully nonlinear Boussinesq equations on generalised curvilinear coordinate systems is proposed. The equations are rearranged in order to solve them by a high resolution hybrid finite volume-finite difference scheme. The conservative part of the above-mentioned equations, consisting of the convective terms and the terms related to the free surface elevation, is discretised by a high-order shockcapturing finite volume scheme; dispersive terms and the term related to the approximation to the second order of the vertical vorticity are discretised by a cell-centred finite difference scheme. The shock-capturing method makes it possible to intrinsically model the wave breaking, therefore no additional terms are needed to take into account the breaking related energy dissipation in the surf zone. The model is applied on a real case regarding the simulation of wave fields and nearshore currents in the coastal region opposite Pescara harbour (Italy).

Keywords: contravariant formulation, Boussinesq equations, breaking waves, run-up, shock-capturing methods.
\end{abstract}




\section{Introduction}

The modelling of surface wave transformation, wave breaking and run-up are of fundamental importance for the simulation of hydrodynamic phenomena which occur in coastal regions. Most of these phenomena can be represented by waveresolving models which are based on the combined solution of the Boussinesq equations for the representation of wave propagation from deep water up to just seaward of the surf zone and the non-linear shallow water equations for the representation of wave propagation in the surf zone (Tonelli and Petti [1]; Shi et al. [2]). These models are able to take into account the non-linear wave-wave interactions, the fully coupled wave-current interactions and the breaking related near shore currents.

Chen [3] improved the representation of the breaking induced coastal circulations by retaining in the motion equations second order terms related to the vertical component of the vorticity.

The modelling of hydrodynamics over computational domains representing the complexity of real case morphologies can be done both by using unstructured grids (Casonato and Gallerano [4]; Cioffi et al. [5]; Gallerano and Napoli [6]; Sørensen et al. [7]) and computational grids obtained by the intersection of boundary conforming coordinate lines. Using curvilinear computational grids the equations can be written in contravariant formulation (Shi et al. [2]; Gallerano and Cannata [8, 9]; Zijlema et al. [10] and Gallerano et al. [11]).

In this paper we present a new Boussinesq type model whose equations are written in a contravariant formulation and solved on curvilinear grids representing the complex morphology of the real case studies. The equations at the base of this model are derived starting from the fully non-linear formulation of the Boussinesq equations proposed by Chen [3] and consistently with this formulation they retain the term related with the second order vertical vorticity. The model is able to represent wave evolution in coastal regions, the breaking phenomenon, the breaking induced longshore and rip currents, the effect of offshore structures on the hydrodynamics, the interaction of the wave motion with the river mouths and run-up hydrodynamics in swash zone.

An upwind WENO scheme for the solution of the equations on generalized curvilinear grids is used in this work. The conservative terms are solved by a high-order finite volume shock-capturing scheme in which an exact Riemann solver is involved.

\section{Conservative form of the Cartesian fully non-linear Boussinesq equations}

Let $H=h+\eta$ be the total local water depth, where $h$ is the local still water depth and $\eta$ is the local surface displacement. Using a Taylor expansion of the velocity about an arbitrary distance from the still water surface, $\sigma$, and assuming zero horizontal vorticity, as proposed by Chen [3], the vertical distribution of the 
horizontal velocity can be written as $\vec{u}=\vec{u}_{\alpha}+\vec{u}_{2}(z)$, where $\vec{u}_{\alpha}$ is the horizontal velocity at an arbitrary distance from the still water level, $z=\sigma$ and $\vec{u}_{2}(z)=(\sigma-z) \nabla\left[\nabla \cdot\left(h \vec{u}_{\alpha}\right)\right]+\left[\left(\sigma^{2} / 2\right)-\left(z^{2} / 2\right)\right] \nabla\left(\nabla \cdot \vec{u}_{\alpha}\right)$ consists of the second order terms in depth power expansion of the velocity vector in which $\nabla$ is the two-dimensional differential operator defined as in a $\nabla=(\partial / \partial x, \partial / \partial y)$ Cartesian reference system.

The following vectors can be defined: $\vec{r}=H \vec{u}_{\alpha}$ and $\vec{s}=H \overrightarrow{\bar{u}}_{2}$, in which $\overrightarrow{\bar{u}}_{2}$ is the depth averaged value of $\vec{u}_{2}(z)$. G is the constant of gravity.

We consider a transformation $x^{l}=x^{l}\left(\xi^{1}, \xi^{2}\right)$ from the Cartesian coordinates $\vec{x}$ to the curvilinear coordinates $\vec{\xi}$ (note that hereinafter the superscript indicates the generic component and not the powers). Let $\vec{g}_{(l)}=\partial \vec{x} / \partial \xi^{l}$ be the covariant base vectors and $\vec{g}^{(l)}=\partial \xi^{l} / \partial \vec{x}$ the contravariant base vectors. The metric tensor and its inverse are defined, respectively, by $g_{l m}=\vec{g}_{(l)} \cdot \vec{g}_{(m)}$ and $g^{l m}=\vec{g}^{(l)} \cdot \vec{g}^{(m)}$ $(l, m=1,2)$. The Jacobian of the transformation is $\sqrt{g}=\sqrt{\operatorname{det}\left(g_{l m}\right)}$. The transformation relationships between the components of the generic vector in the Cartesian coordinate system and its contravariant and covariant components, $b^{l}$ and $b_{l}$, in the curvilinear coordinate system are given by $b^{l}=\vec{g}^{(l)} \cdot \vec{b}, \vec{b}=b^{l} \vec{g}_{(l)}, b_{l}=\vec{g}_{(l)} \cdot \vec{b}, \vec{b}=b_{l} \vec{g}^{(l)}$. In the following equations, a comma with an index in a subscript stands for covariant differentiation. The covariant derivative is defined as $b^{l}{ }_{m}=\partial b^{l} / \partial \xi^{m}+\Gamma_{m k}^{l} b^{k}$, where $\Gamma_{m k}^{l}$ is the Christoffel symbol (Aris [12]) given by $\Gamma_{m k}^{l}=\vec{g}^{(l)} \cdot \partial \vec{g}_{(k)} / \partial \xi^{m}$.

Let $r^{* l}$ be the $l-t h$ contravariant component of a vector $\vec{r}^{*}$ defined by $r^{* l}=r^{l}+H V^{l}$. Let $\Delta A$ be the area of the generic surface element and $\overrightarrow{\tilde{g}}^{(l)}=\vec{g}^{(l)}\left(\xi_{0}^{1}, \xi_{0}^{2}\right)$ the contravariant base vector defined at point $P_{0} \in \Delta A$ whose coordinates are $\xi_{0}^{1}$ and $\xi_{0}^{2}$. Let also be $\lambda_{k}\left(\xi^{1}, \xi^{2}\right)=\overrightarrow{\tilde{g}}^{(l)} \cdot \vec{g}_{(k)}$ the covariant component of $\overrightarrow{\tilde{g}}^{(l)}$ where $\vec{g}_{(k)}=\vec{g}_{(k)}\left(\xi^{1}, \xi^{2}\right)$. The integral contravariant form of the continuity and momentum can be expressed as

$$
\begin{gathered}
\iint_{\Delta A} \frac{\partial H}{\partial t} d A+\int_{L} r^{m} n_{m} d L=\iint_{\Delta A}\left(s^{l}\right)_{, l} d A \\
\iint_{\Delta A} \overrightarrow{\tilde{g}}^{(l)} \cdot \vec{g}_{(k)} \frac{\partial r^{* k}}{\partial t} d A+\int_{L}\left(\overrightarrow{\tilde{g}}^{(l)} \cdot \vec{g}_{(k)} \frac{r^{k} r^{m}}{H}+G \overrightarrow{\tilde{g}}^{(l)} \cdot \vec{g}^{(m)} \frac{H^{2}}{2}\right) n_{m} d L=\iint_{\Delta A} \overrightarrow{\tilde{g}}^{(l)} \cdot \vec{g}_{(k)} G H g^{k m} h_{, m} d A \\
-\iint_{\Delta A} \overrightarrow{\tilde{g}}^{(l)} \cdot \vec{g}_{(k)} R^{k} d A-\iint_{\Delta A} \overrightarrow{\tilde{g}}^{(l)} \cdot \vec{g}_{(k)} \frac{r^{k}}{H}\left(s^{m}\right)_{, m} d A+\iint_{\Delta A} \overrightarrow{\tilde{g}}^{(l)} \cdot \vec{g}_{(k)} \frac{\partial H}{\partial t} V^{k k} d A \\
-\iint_{\Delta A} \overrightarrow{\tilde{g}}^{(l)} \cdot \vec{g}_{(k)} H V^{\prime \prime k} d A-\iint_{\Delta A} \overrightarrow{\tilde{g}}^{(l)} \cdot \vec{g}_{(k)} H T^{k} d A-\iint_{\Delta A} \overrightarrow{\tilde{g}}^{(l)} \cdot \vec{g}_{(k)} H W^{k} d A
\end{gathered}
$$


where $\mathrm{L}$ is the contour line of $\Delta A$ and $n_{m}$ is the $m$-th component of the covariant outward normal. In eqn. (1) the second term on the left-hand side is the flux term. In eqn. (2) the second term on the left hand side is the flux term, the first term on the right hand side is the source term related to the bottom slope, the second term on the right hand side, $R^{l}$, is the bottom resistance term. Expressions for terms $s^{l}, V^{l}, V^{\prime l l}, T^{l}$ and $W^{l}$ are given by

$$
\begin{gathered}
s^{l}=(h+\eta)\left\{\left[\frac{\sigma^{2}}{2}-\frac{1}{6}\left(h^{2}-h \eta+\eta^{2}\right)\right] g^{l m}\left[\left(\frac{r^{k}}{H}\right)_{, k}\right]_{, m}+\left[\sigma+\frac{1}{2}(h-\eta)\right] g^{l m}\left[\left(h \frac{r^{k}}{H}\right)_{, k}\right]_{, m}\right\} \\
V^{l}=\frac{1}{2} \sigma^{2} g^{l m}\left[\left(\frac{r^{k}}{H}\right)_{, k}\right]_{, m}+\sigma g^{l m}\left[\left(h \frac{r^{k}}{H}\right)_{, k}\right]_{, m}-g^{l m}\left[\frac{1}{2} \eta^{2}\left(\frac{r^{k}}{H}\right)_{, k}+\eta\left(h \frac{r^{k}}{H}\right)_{, k}\right]_{, m} \\
T^{l}=g^{l m}\left\{(\sigma-\eta)\left(\frac{r^{i}}{H}\left[\left(h \frac{r^{k}}{H}\right)_{, k}\right]_{, i}\right) g^{l m}\left[\frac{\partial}{\partial t}\left(\frac{\eta^{2}}{2}\right)\left(\frac{r^{k}}{H}\right)_{, k}\right]_{, m}+g^{l m}\left[\frac{\partial \eta}{\partial t}\left(h \frac{r^{k}}{H}\right)_{, k}\right]_{, m}\left(\frac{r^{i}}{H}\left[\left(\frac{r^{k}}{H}\right)_{, k}\right]_{, i}\right)\right\}_{, m}+\frac{1}{2} g^{l m}\left\{\left[\left(h \frac{r^{k}}{H}\right)_{, k}+\eta\left(\frac{r^{k}}{H}\right)_{, k}\right]_{, m}^{2}\right\} \\
W^{l}=\left(\varepsilon^{m i} g_{i p} \frac{r_{m}^{p}}{H}\right) \varepsilon^{j l} \frac{s_{j}}{H}+\left(\varepsilon^{m i} g_{i p} \frac{s_{, m}^{p}}{H}\right) \varepsilon^{j l} \frac{r_{j}}{H}
\end{gathered}
$$

where

$\varepsilon^{m i}=\frac{1}{\sqrt{g}}$ if $(m, i)$ is an even permutation of $(1,2) ;-\frac{1}{\sqrt{g}}$ if $(m, i)$ is an odd permutation of $(1,2) ; 0$ if the two indices are equal.

The eqns (1) and (2) represent the integral expressions of the fully non-linear Boussinesq equations in contravariant formulation in which Christoffel symbols are absent. These equations are accurate to $O\left(\mu^{2}\right)$ and $O\left(\varepsilon \mu^{2}\right)$ in dispersive terms and retain the conservation of potential vorticity up to $O\left(\mu^{2}\right)$, in accordance with the formulation proposed by Chen [3].

The eqns (1) and (2) are solved by a hybrid finite volume-finite difference scheme. Convective terms and terms related to the free surface elevation gradient are discretized by a high order finite volume upwind WENO scheme; dispersive terms and the term related to the second order vertical vorticity are discretized by a finite-difference scheme. The upwind WENO scheme needs a flux calculation at the cell interfaces. These fluxes are calculated by means of the solution of a Riemann problem. An exact Riemann solver is used in this work. No additional dissipative term to improve the modelling of breaking related energy decay and breaking induced near shore circulation is used in this paper. 


\section{The numerical scheme}

The numerical integration of eqns (1) and (2) is carried out by a high order upwind WENO hybrid finite volume-finite difference scheme. The conservative part of the above-mentioned equations, consisting of the convective terms and the terms related to the free surface elevation, is discretized by a high-order shock-capturing finite volume scheme; dispersive terms and the term related to the approximation to the second order of the vertical vorticity are discretized by a cell-centred finite difference scheme. The shock-capturing method makes it possible to intrinsically model the wave breaking, therefore no additional terms are needed to take into account the breaking related energy dissipation in the surf zone.

The computational domain discretization is based on a grid defined by the coordinate lines $\xi^{1}$ and $\xi^{2}$ and by the points of coordinates $\xi_{i}^{1}=i \Delta \xi^{1}$ and $\xi_{j}^{2}=j \Delta \xi^{2}$, which represent the centres of the calculation cells $I_{i, j}=\left(\xi_{i-1 / 2}^{1}, \xi_{i+1 / 2}^{1}\right) \times\left(\xi_{i-1 / 2}^{2}, \xi_{i+1 / 2}^{2}\right) . t^{n}$ is the time level of the known variables, while $t^{n+1}=t^{n}+\Delta t$ is the time level of the unknown variables. Let us indicate with $L\left(r^{1}, r^{2}\right)$ and with $L_{B}\left(s^{1}, s^{2}\right)$ respectively the first and the second term on the right hand side of eqn. (13). Let us indicate with $\boldsymbol{D}\left(H, r^{1}, r^{2}\right)$ the numerical approximation of the sum of the convective and free surface elevation terms (which is split in order to ensure a well-balanced scheme) on the right hand side of eqn. (2) and with $D_{B}\left(H, r^{1}, r^{2}, s^{1}, s^{2}\right)$ the bottom friction term, the numerical approximation of the sum of dispersive terms and the term related to the approximation to the second order of the vertical vorticity on the right-hand side of this equation. By integrating eqns (1) and (2) over $\left[t^{n}, t^{n+1}\right]$ we get

$$
\begin{gathered}
\tilde{\bar{H}}_{i ; j}^{(n+1)}=\tilde{\bar{H}}_{i ; j}^{(n)}-\frac{1}{\Delta A} \int_{t^{n}}^{t^{n+1}}\left[\boldsymbol{L}\left(r^{1}, r^{2}\right)+\boldsymbol{L}_{B}\left(s^{1}, s^{2}\right)\right] d t \\
\tilde{\bar{r}}_{i ; j}^{* l(n+1)}=\tilde{\bar{r}}_{i, j}^{* t(n)}-\frac{1}{\Delta A} \int_{t^{n}}^{t^{n+1}}\left[\boldsymbol{D}\left(H, r^{1}, r^{2}\right)+\boldsymbol{D}_{B}\left(H, r^{1}, r^{2}, s^{1}, s^{2}\right)\right] d t
\end{gathered}
$$

Eqns (8) and (9) represent the advancing from time level $t^{n}$ to time level $t^{n+1}$ of the variables $\tilde{\bar{H}}_{i, j}$ and $\tilde{\bar{r}}_{i, j}^{*}$. The state of the system is known at the centre of the calculation cell and it is defined by the cell-averaged values $\tilde{\bar{H}}_{i ; j}$ and $\tilde{\bar{r}}_{i, j}^{*}$. In this paper, time integration of eqns (8) and (9) is carried out by means of a third order accurate Strong Stability Preserving Runge-Kutta method (SSPRK) reported in Spiteri and Ruuth [13]. The SSPRK method can be written in compact form as follows

$$
\begin{gathered}
\tilde{\bar{H}}_{i, j}^{(0)}=\tilde{\bar{H}}_{i, j}^{(n)} ; \tilde{\bar{r}}_{i ; j}^{*}(0)=\tilde{\bar{r}}_{i ; j}^{*(n)} \\
\tilde{\bar{H}}_{i ; j}^{(p)}=\sum_{q=0}^{p-1}\left\{\Omega_{p q} H_{i ; j}^{(q)}+\Delta t \varphi_{p q}\left[L\left(r^{1(q)}, r^{2(q)}\right)+L_{B}\left(s^{1(p)}, s^{2(p)}\right)\right]\right\} \\
\tilde{\bar{r}}_{i, j}^{*(p)}=\sum_{q=0}^{p-1}\left\{\Omega_{p q} r_{i ; j}^{*(q)}+\Delta t \varphi_{p q}\left[D\left(H^{(q)}, r^{1(q)}, r^{2(q)}\right)+D_{B}\left(H^{(p)}, r^{1(p)}, r^{2(p)}, s^{1(p)}, s^{2(p)}\right)\right]\right\} \\
\tilde{\bar{H}}_{i ; j}^{(n+1)}=\tilde{\bar{H}}_{i ; j}^{(3)} ; \tilde{\bar{r}}_{i, j}^{*(n+1)}=\tilde{\bar{r}}_{i, j}^{* l(3)}
\end{gathered}
$$


where $p=1 ; 2 ; 3$. See Spiteri and Ruuth [13] for $\Omega_{p q}$ and $\varphi_{p q}$ values. The computation of $\boldsymbol{L}\left(r^{1}, r^{2}\right), \boldsymbol{D}\left(H, r^{1}, r^{2}\right), \boldsymbol{L}_{B}\left(s^{1}, s^{2}\right)$ and $\boldsymbol{D}_{B}\left(H, r^{1}, r^{2}, s^{1}, s^{2}\right)$ terms needs the numerical approximation of the spatial integrals on the right-hand side of eqns (1) and (2). This numerical approximation is carried out by means of a hybrid finite volume-finite difference scheme as used by Shi et al. [2]. By applying this method, once the values of the auxiliary variable $\tilde{\tilde{r}}^{*}$ are known, the values of the original variables $\tilde{\bar{r}}^{l}$ at each stage of the Runge-Kutta method are computed solving the following equation

$$
\tilde{\bar{r}}^{* l}=\tilde{\bar{r}}^{l}+\tilde{\bar{H}} \tilde{\bar{V}}^{l}
$$

in which $\tilde{\bar{V}}^{\prime l}$ includes first and second derivative of $\tilde{\bar{r}}^{l} / \tilde{\bar{H}}$ with respect to $\xi^{1}$ and to $\xi^{2}$ and cross derivatives. The numerical approximation of the derivatives in the term is carried out by a second order central difference scheme. The velocity at the elevation $\sigma$, averaged over the generic computational cell $I_{i ; j}$ and indicated with $\left(\tilde{\bar{r}}^{l} / \tilde{\bar{H}}\right)_{i ; j}$ can be found by solving a system of equations with tridiagonal matrix formed by eqn. (14) in which all cross-derivatives are moved to the right hand side of the equation.

Once the values of $\left(\tilde{\bar{r}}^{l} / \tilde{\bar{H}}\right)_{i ; j}$ are known, the $L_{B}\left(s^{1}, s^{2}\right)$ and $\boldsymbol{D}_{B}\left(H, r^{1}, r^{2}, s^{1}, s^{2}\right)$ terms on the right hand side of eqns (11) and (12) are discretized using a second order central difference scheme at the cell centroids. Since the $L_{B}$ and $D_{B}$ terms need to be updated using $H, r^{1}, r^{2}, s^{1}, s^{2}$ at the corresponding time step, an iteration is needed to achieve convergence, as suggested by Shi et al. [2]. Convective terms and terms related to the free surface elevation that define the $\boldsymbol{L}\left(r^{1}, r^{2}\right)$ and $\boldsymbol{D}\left(H, r^{1}, r^{2}\right)$ terms on the right-hand side of eqns (11) and (12) are computed by a high-order finite volume WENO scheme, according to the procedure proposed by Gallerano et al. [14].

A wet and dry technique is used to catch moving shoreline: if the water depth at the center of the computational cell is greater than a given threshold value the fluxes at the cell interfaces are computed using the wet bed solution of the Riemann problem, otherwise the fluxes at the cell interfaces are computed using the dry bed solution of the Riemann problem (Toro [15]).

\section{Results}

The capacity of the proposed model to correctly represent wave propagation, wave breaking and wave induced currents in curvilinear boundary conforming grids has been verified against test cases present in literature (Gallerano et al. [11]). In this section the model is applied to a real case regarding the simulation of the wave fields and wave induced nearshore currents in the coastal region opposite Pescara harbour (Italy). 


\subsection{Wave field and nearshore currents simulation in the Pescara coastal area}

The port of Pescara is located on the Italian Adriatic coast at the mouth of the homonymous river. It was established at the end of the nineteenth century as an inland port for the local fishing fleet; the inner basin of this port is located in the final stretch of the river Pescara.

In fig. 1A the plan view of the port areas without the outer port structures is outlined. Fig. 2 shows the bottom geometry of the coastal area in front the port of Pescara. In fig. 1B the plan view of the port areas with the outer port structures built in the 90 s is outlined.

The area is affected by significant longshore currents. The incident wave motion interacts with both the waves reflected and diffracted by the port structures (wave-wave interactions) and with the river flow at the entrance of the inland port (wave-current interaction).

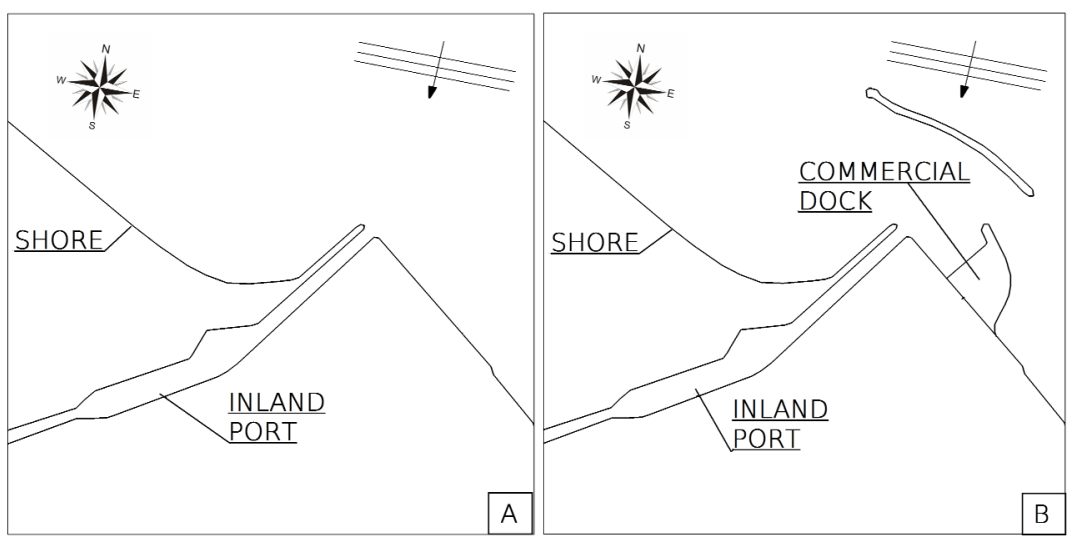

Figure 1: Wave field and nearshore currents simulation in the Pescara coastal area. Plan view of the Pescara port: A) Absence of outer port structures. B) Presence of outer port structures.

Due to the complexity of the morphological and hydrodynamic phenomena that occur, wave fields and nearshore currents can be simulated only by means of wave-resolving models based on the combined solution of the Boussinesq equations for the representation of the motion equation from deep water up to just seaward of the surf zone and the nonlinear shallow water equations for the representation of the wave motion in the surf zones.

The contravariant formulation of the equations presented in this work, permits the numerical integration of the above mentioned equations on generalized curvilinear grids representing the complex morphology of the considered coastal area. The proposed model takes into account the complexity of the hydrodynamic phenomena taking place in the area in front of the port of Pescara 


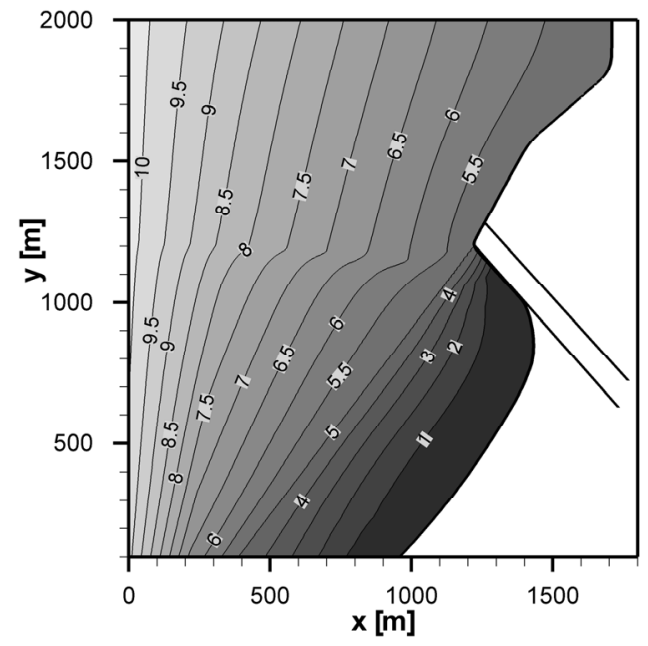

Figure 2: Wave field and nearshore currents simulation in the Pescara coastal area. Two-dimensional representation of bottom geometry of the coastal area in front of the Pescara harbour.

and consisting in non-linear wave-wave interactions between the incoming wave motion and the waves reflected and diffracted around the structures, fully coupled wave-current interactions between the wave motion and the river current and coastal circulations induced by the wave breaking.

The equations at the basis of this model are discretized on a boundary conforming curvilinear grid. At the coastline, swash hydrodynamic phenomena were simulated using a procedure that involves a Riemann problem solver relative to a wet-dry front. In fig. 3A is shown the instantaneous surface elevation produced by waves of a deep water height of $1.5 \mathrm{~m}$ with propagation direction oriented from North to South. From the figure analysis it can be seen how the wave the refraction, shoaling and reflection alter the wave field close to the coastline. Close to the river mouth, to its East, reflection of the incoming wave motion can be seen.

The interaction of the wave motion with the reflected waves and the river current, which is associated with a discharge of $300 \mathrm{~m}^{3} / \mathrm{s}$, produces an increase of the wave height values of up to $1.98 \mathrm{~m}$.

In fig. 3B is shown the time and depth-averaged velocity field associated with the wave field presented in fig. 3A and with a river discharge of $300 \mathrm{~m}^{3} / \mathrm{s}$. The aforesaid velocity fields show the presence of a longshore current flowing parallel with the coastline and a vortex to the West of the entrance of the inland port.

In fig. 4A is shown the instantaneous free surface elevation produced by waves of deep water height of $1.5 \mathrm{~m}$ and the direction of propagation oriented from North to South in the presence of the offshore breakwater and the freight docks. From the analysis of fig. 4A the diffraction phenomena can also be seen around the ends of the offshore breakwater. This breakwater drastically reduces 

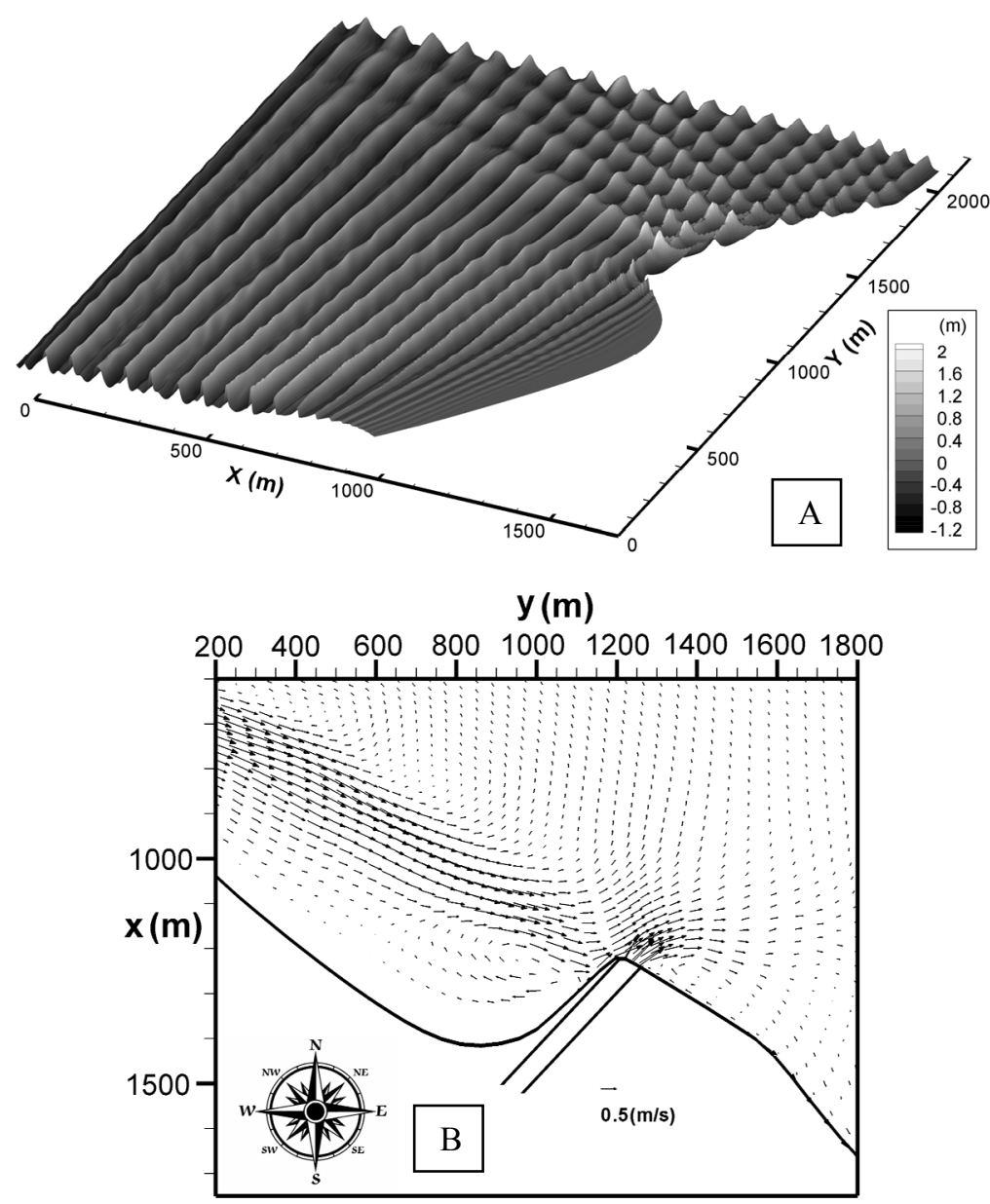

Figure 3: Wave field and nearshore currents simulation in the Pescara coastal area. A) Wave field in absence of port structures. B) Nearshore currents in absence of port structures. Average velocity values in absence of port structures. River discharge: $300 \mathrm{~m}^{3} / \mathrm{s}$.

the wave height, modifies the wave front because of the wave diffraction and induces the reflection phenomena seaward of the breakwater.

In the area between the offshore breakwater and the entrance of the inland port the wave energy, dissipated by the presence of the structures, is considerably reduced: the wave height values are never higher than $0.2 \mathrm{~m}$.

In fig. 4B is shown the time and depth-averaged velocity field produced by the wave field presented in fig. $4 \mathrm{~A}$ and by a river discharge of $300 \mathrm{~m}^{3} / \mathrm{s}$. 

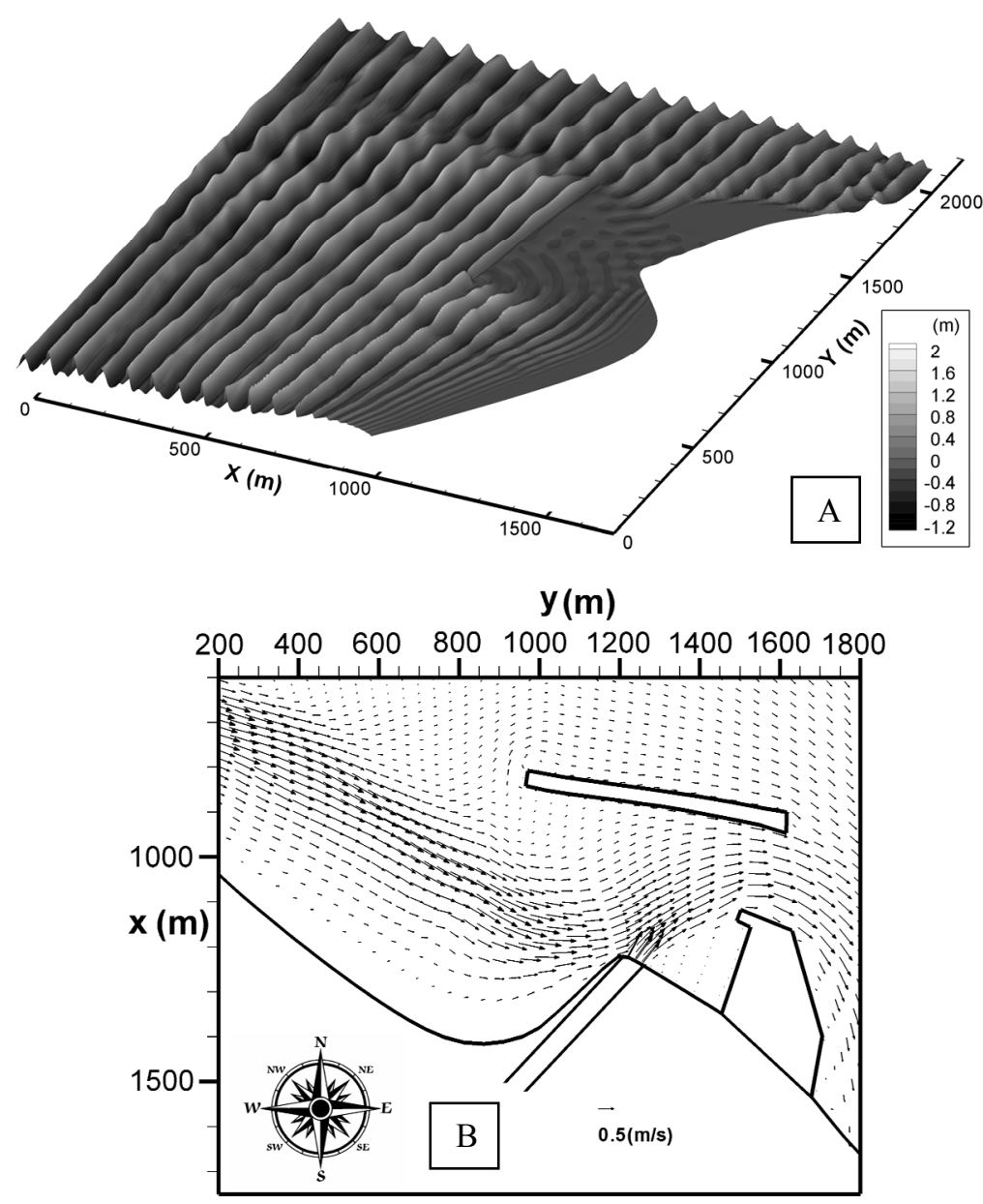

Figure 4: Wave field and nearshore currents simulation in the Pescara coastal area. A) Wave field in presence of port structures. B) Nearshore currents in presence of port structures. Average velocity values in absence of port structures. River discharge: $300 \mathrm{~m}^{3} / \mathrm{s}$.

From the analysis of fig. 4B the absence of the vortex can be noted which instead is present in the velocity field relative to the absence of the port structures shown in fig. 3B. From fig. 4B it can also be observed that the longshore current, induced by the breaking of the waves on the sandy beach, is channelled into the area between the mouth of the river Pescara and the outer port structures. The aforesaid current has the same order of magnitude as the one occurring in the absence of the outer port structures as shown in fig. $3 \mathrm{~B}$.

The analysis of hydrodynamics fields can provide useful information for a qualitative assessment around the way in which the break water and docks, changing substantially the wave field and the velocity field of the coastal current, 
modify transport conditions and material storage in the area in front of the mouth of the river and induce a change in the seabed morphology.

\section{Conclusions}

In this paper a contravariant formulation of the fully non-linear Boussinesq equations has been proposed in order to simulate wave transformation phenomena, wave breaking and run-up in computational domains representing the complex morphology of real coastal regions.

The motion equations include the term related to the approximation to the second order of the vertical vorticity. A new Upwind Weighted Essentially NonOscillatory scheme for the solution of the fully nonlinear Boussinesq equations on generalized curvilinear coordinate systems has been proposed. A high order shock capturing method in which an exact Riemann solver is involved has been used to intrinsically model the wave breaking.

The model has been applied to a real case regarding the simulation of wave fields and nearshore currents in the coastal region opposite Pescara harbour (Italy).

\section{References}

[1] Tonelli M. \& Petti M., Hybrid finite volume-finite difference scheme for 2DH improved Boussinesq equations. Coastal Engineering, 56(5-6), pp. 609-620, 2009.

[2] Shi F., Kirby J.T., Harris J.C., Geiman J.D. \& Grilli S.T., A high-order adaptive time-stepping TVD solver for Boussinesq modeling of breaking waves and coastal inundation. Ocean Modelling, 43, pp. 36-51, 2012.

[3] Chen, Q., Fully nonlinear Boussinesq-type equations for waves \& currents over porous beds. Journal of Engineering Mechanics, 132(2), pp. 220 230, 2006.

[4] Casonato M. \& Gallerano F., A finite difference self-adaptive mesh solution of flow in a sedimentation tank. International Journal For Numerical Methods In Fluids, 10(6), pp. 697-711, 1990.

[5] Cioffi F.; Gallerano F. \& Napoli E., Three-dimensional numerical simulation of wind driven flows in closed channels and basins. Journal of Hydraulic Research, 43(3), pp. 290-301, 2005.

[6] Gallerano F., Napoli E., A dynamic subgrid-scale tensorial Eddy viscosity model. Continuum Mech. Thermodyn, 11(1), pp. 1-14, 1999.

[7] Sørensen O.R., Schäffer H.A. \& Sørensen L.S., Boussinesq-type modeling using an unstructured finite element technique. Coastal Engineering, 50(4), pp. 181-198, 2004.

[8] Gallerano F. \& Cannata G., Compatibility between reservoir sediment flushing and river protection. Journal of Hydraulic Engineering, 137 (10), pp. 1111-1125, 2011. 
[9] Gallerano F. \& Cannata G., Central WENO scheme for the integral form of contravariant shallow-water equations. International Journal For Numerical Methods In Fluids, 67(8), pp. 939-959, 2011.

[10] Zijlema M., Segal A. \& Wesseling P., Invariant discretization of the $\mathrm{k}-\varepsilon$ model in general co-ordinates for prediction of turbulent flow in complicated geometries. Computers \& Fluids, 24(3), pp. 209-225, 1995.

[11] Gallerano F., Cannata G. \& Villani M., An Integral Contravariant Formulation Of The Fully Non-Linear Boussinesq Equations, Coastal Engineering, 83, pp. 119-136, 2014.

[12] Aris R., Vectors, Tensors, and the Basic Equations of Fluid Mechanics. New York: Dover Book on Mathematics, 320, 1989.

[13] Spiteri R.J. \& Ruuth S.J., A new class of optimal high-order strongstability preserving time discretization methods. SIAM Journal on Numerical Analysis, 40(2), pp. 469-491, 2002.

[14] Gallerano F.; Cannata G. \& Tamburrino M., Upwind WENO scheme for Shallow Water Equations in contravariant formulation. Computers \& Fluids, 62, pp. 1-12, 2012.

[15] Toro E., Shock-Capturing Methods for Free-Surface Shallow Flows. Manchester: John Wiley and Sons, 2001. 